\title{
The entrainment of EEG delta oscillations in speech listening
}

\author{
Victor J. Boucher ${ }^{1}$, Annie C. Gilbert ${ }^{2}$, Boutheina Jemel ${ }^{3}$ \\ ${ }^{1}$ Département de linguistique, Université de Montréal, Canada \\ ${ }^{2}$ School of Communication Sciences and Disorders, McGill University, Canada \\ ${ }^{3}$ Département d'audiologie et orthophonie, Université de Montréal, Canada \\ https://doi.org/10.36505/ExLing-2017/08/0007/000309
}

\begin{abstract}
Research on the sensory entrainment of neural oscillations provides a novel way of understanding how the brain processes spoken language without postulates of interim linguistic units. Several reports have shown that oscillations in the theta range $(3-10 \mathrm{~Hz})$ are entrained by syllable-size modulations in the energy envelope of speech. This entrainment has recently been shown to provide sensory frames in processing feature-related cues. A similar perspective has been applied to delta waves $(<3 \mathrm{~Hz})$. Thus, it is suggested that delta entrains to long ("sentence"-size) energy contours, and this would provide processing frames involved in utterance comprehension. The present paper adopts a different viewpoint based on our previous work showing that delta-size perceptual chunks in speech relate to an online sensory memory of sequential information. We used electro-encephalography (EEG) to monitor listeners $(n=18)$ on-line responses to utterance stimuli with controlled patterns of energy, pitch, and temporal marks. Measures of inter-trial phase coherence in neural oscillations show that delta specifically entrains to temporal chunks in the stimuli. This supports a view that delta is not entrained by sentence-size patterns but by perceptual chunks which provide sensory frames in processing incoming sequential information in heard speech.
\end{abstract}

Key words: neural oscillations, entrainment, perceptual chunking, sensory memory

\section{Introduction - The role of neural entrainment}

Experimentalists who refer to linguistics in elaborating tests with speech stimuli are incessantly confronted to the problem that units of linguistic analyses do not directly reflect in signals. Separate letter-like "phonemes" are not observed in speech acoustics or physiology. It is also acknowledged, that there are no operational definitions of "words" and "sentences" -- except by reference to spaces in text (e.g. Haspelmath, 2011), and a fortiori there are no consistent marks for dividing such units across contexts and languages. Finally, to this day, one finds no evidence in neurosciences that supports a processing of utterances in terms of hierarchical combinations of phonemes, words, and sentences, as theorized in formal linguistics (Ingram, 2007). On this problem of directly linking brain processes to speech signals, research on neural entrainment provides a new perspective.

ExLing 2017: Proceedings of 8th Tutorial and Research Workshop on Experimental Linguistics, 19-22 June, Heraklion, Crete, Greece 
In particular, studies have shown that neural oscillations in the theta range $(3-10 \mathrm{~Hz})$ are entrained by syllable-size modulations in the energy envelope of continuous speech. This entrainment, often measured in terms a "cerebroacoustic coherence", creates theta-length sensory windows which are involved in processing "distinctive features" (e.g. Luo \& Poeppel, 2012). To illustrate how this functions, one can refer to temporal indices in speech such as voice onset time (VOT) and transitions, which cue voice and place features (e.g. $/ \mathrm{ta} /-/ \mathrm{da} /, / \mathrm{ta} /-/ \mathrm{ka} /$ ), respectively. It is known that these temporal indices vary with speech rate in that VOTs and transitions shorten when speaking at fast rates. Yet listeners correctly categorize sounds, basically because the indices are perceived relative to a syllable frame. This is seen in Fig. 1: absolute ms values of VOT vary with speech rate (panel A) but are categorical when one measures VOT as a ratio relative to syllable durations (panel B). In fact, altering the perceptual frame of syllable cycles, without modifying VOT, can influence categorization (Boucher, 2002).

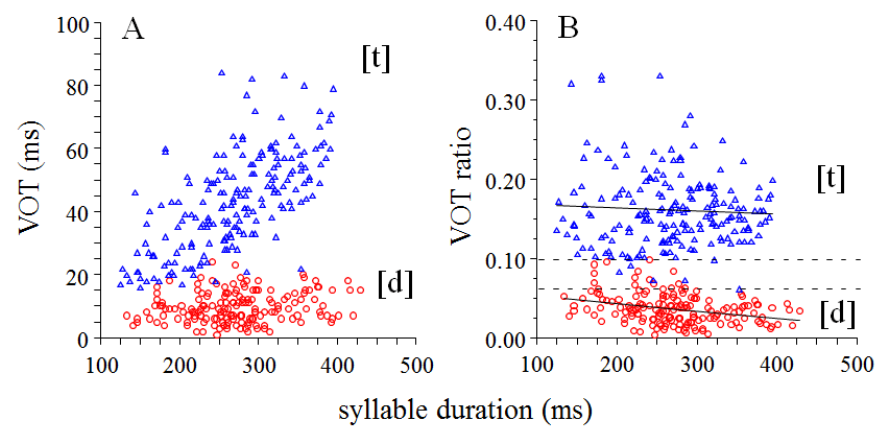

Figure 1. Variations of VOT across speech rates. A-absolute VOTs; Brelative VOTs. A category boundary supporting distinctions is given by $\mathrm{B}$.

A recent brain-imaging study has illustrated this effect in terms of neural entrainment. Thus, ten Oever and Sack (2015) showed that entraining theta oscillations on varying syllable durations can influence the categorization of transitions. In short, indices that cue classical feature distinctions are processed in terms of a sensory frame that links directly to theta waves, and there is no need in this approach to postulate interim units of representation like phonemes or groups of consonants and vowels.

As for longer frames, some research suggests that energy envelopes variably related to prosodic groups or "sentences" can provide processing frames that bear on utterance "comprehension" (e.g., Park et al. 2015). In these reports, however, cerebro-acoustic coherence was measured by reference to energy contours of continuous speech. Consequently, the contours could reflect a number of structures in speech. In our approach, we 
used utterance stimuli with narrowly controlled pitch, intensity, and temporal patterns so as to identify the effects of given structures on brain responses. We found that an on-line perceptual chunking operates specifically in terms of temporal groups, and presents effects on the sensory memory of heard items in utterances, whether these contain meaningful or incomprehensible syllables (Gilbert et al, 2014, 2015). Hence, the perceptual chunking did not relate to utterance comprehension per se, but to a memory processing of incoming sequential information. Moreover, in examining listeners' average brain responses to the stimuli, group-related periodicities appeared that could match oscillations in the delta range - as illustrated in Fig. 2. In order to verify whether such patterns in EEG reflected the entrainment of delta, we performed the present experiment.

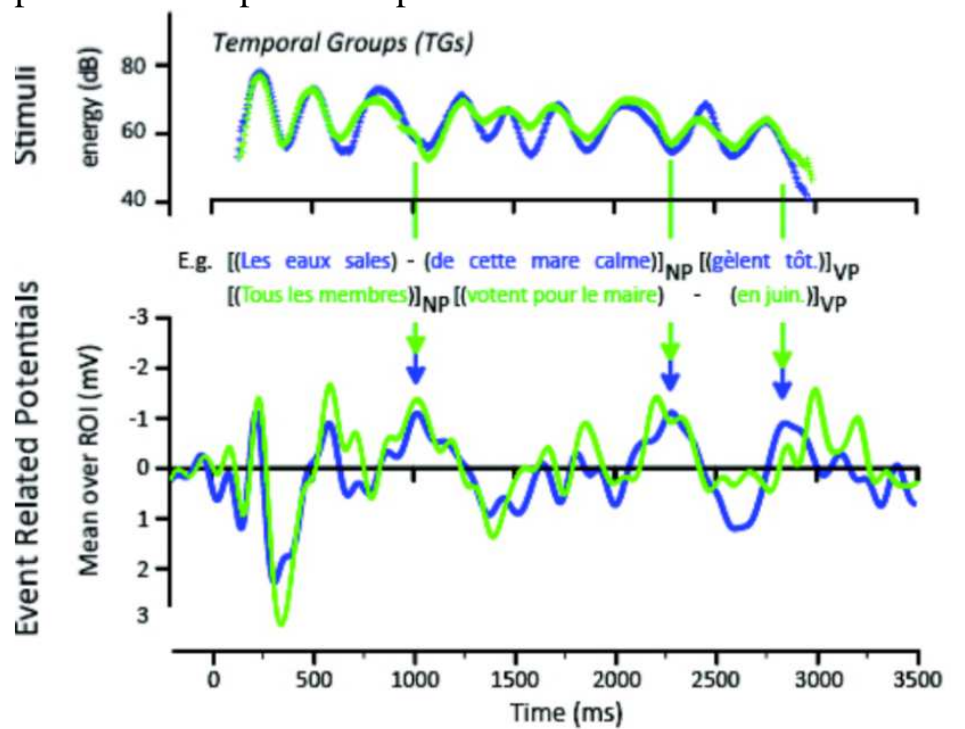

Figure 2. Listeners' ERPs $(n=20)$ at electrode $\mathrm{Cz}$ follow temporal groups in utterances (50 trials per condition), regardless of whether intonation contours reset on the third (green) or seventh (blue) syllable (from Gilbert et al. 2015)

\section{Method and results -- Delta entrainment}

Eighteen listeners heard utterances containing regular temporal groups at periods of $685 \mathrm{~ms}(1.46 \mathrm{~Hz})$, with narrowly controlled intonation and energy envelopes. Stimuli construction and EEG recording was that of previous studies of Gilbert et al $(2014,2015)$. In the analyses, however, we calculated the inter-trial phase coherence (ITPC) using EEGLAB (Delorme \& Makeig, 2004), which measures how brain oscillations align with the heard stimuli at different frequencies. Fig. 3 presents a time-frequency plot of ITPC. One can see delta-range oscillations at about $1.5 \mathrm{~Hz}$ elicited while participants heard 
utterances with regular temporal groups of $1.46 \mathrm{~ms}$. Hence the delta-range response was specifically evoked by the periodicities of temporal groups.

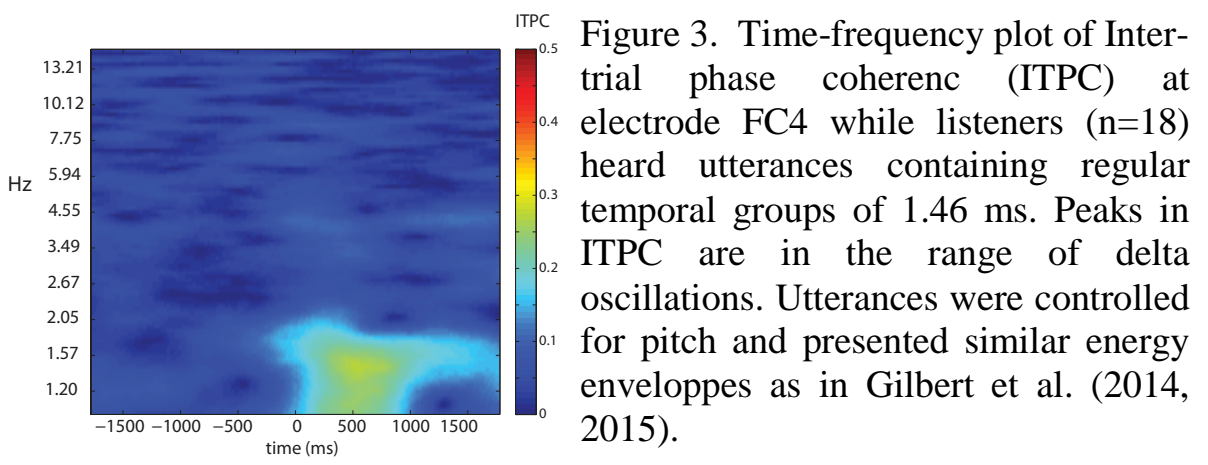

\section{Discussion and prospective}

The above entrainment was also observed with meaningless series of syllables and, consequently is not involved in sentence comprehension, as suggested by Park et al. (2015). However, delta entrainment was especially clear at fronto-central sites, and this, in conjunction with earlier findings, suggests that delta oscillations may relate to perceptual chunks involved in processing sequential sensorimotor information. Further analyses comparing listeners' responses to speech and non-speech will serve to confirm whether delta entrainments bears specifically on a processing of sensorimotor aspects as opposed to a processing of acoustic information.

\section{References}

Boucher, V.J. 2002. Timing relations in speech and the identification of voice-onset times. Perception \& Psychophysics 64, 121-130.

Gilbert, A.C., Boucher, V.J., Jemel, B. 2015. The perceptual chunking of speech: A demonstration using ERPs. Brain Research 1603, 101-113.

Gilbert, A.C., Boucher, V.J., Jemel, B. 2014 Perceptual chunking and its effect on memory in speech processing. Frontiers in Psychology 5,1-9.

Haspelmath, M. 2011. The indeterminacy of word segmentation and the nature of morphology and syntax. Folia Linguistica 45, 31-80.

Ingram, J.C.L. 2007. Neurolinguistics. An introduction to spoken language processing and its disorders. Cambridge UK: Cambridge University Press.

Luo, H., Poeppel, D. 2012 Cortical oscillations in auditory perception and speech... Frontiers in Psychology 3,170.

Park, H. et al 2015 Frontal top-down signals increase coupling of auditory lowfrequency oscillations. Current Biology 25, 1649-1653.

Ten Oever, S., Sack, A.T. 2015 Oscillatory phase shapes syllable perception. P. N. A. S. 112, 15833-15837. 